\title{
IMPROVEMENT OF COLLABORATION BETWEEN TESTING AND SIMULATION DEPARTMENTS ON THE EXAMPLE OF A MOTORCYCLE MANUFACTURER
}

\author{
Schönwald, Julian Ralf (1); Forsteneichner, Christian (2); Vahrenhorst, David (1); Paetzold, \\ Kristin (1) \\ 1: Universität der Bundeswehr München; 2: BMW Motorrad
}

\begin{abstract}
In testing and simulation departments in product development (PD) data types, data structures and data storage are often very different. Exchange of data and information is normally not automated and often not supported by management systems. This can lead to loss of time and information. A literature study in combination with 20 expert interviews and the analysis of documents as well as data storage structures and IT systems in a PD department of a motorcycle manufacturer were performed. Test and simulation processes were classified and standardized, documentation formats analyzed, standards in Test Data Management (TDM) and Simulation Data Management (SDM) as well as verification and validation processes compared. IT support in SDM is better than in TDM. An integration of TDM and SDM could lead to improved collaboration between testing and simulation departments. Options for this integration could be specific ontologies, object-oriented interfaces, a higher-level intermediate application, use of a common standard or integration of one standard into another one.
\end{abstract}

Keywords: Testing, Simulation, Case study, Collaborative design, Data Management

Contact:

Schönwald, Julian Ralf

Universität der Bundeswehr München

Institute for technical product development

Germany

julian.schoenwald@unibw.de

Cite this article: Schönwald, J.R., Forsteneichner, C., Vahrenhorst, D., Paetzold, K. (2019) 'Improvement of

Collaboration between Testing and Simulation Departments on the Example of a Motorcycle Manufacturer', in

Proceedings of the 22nd International Conference on Engineering Design (ICED19), Delft, The Netherlands, 5-8 August

2019. DOI:10.1017/dsi.2019.18 


\section{INTRODUCTION}

Nowadays high quality requirements, homologation as well as implementation of new technologies and materials in existing systems lead to large uncertainties and high risks in product development. Verification and validation is indispensable in the product development process (PDP). It is needed to understand physical relationships between components and to reduce the risk of mistakes during the development process.

In the early years of industrial production of automobiles cars showed many mistakes and weaknesses which lead to breakdowns or accidents. As a consequence, it was started to physically test them at the beginning of the 20th century. The first test methods were developed - mainly in the field of material and component testing. These were refined and supplemented in the course of time. The tasks became more varied (e.g. property assurance, determination of characteristic values, support of validation). In the last decades a new discipline for verification and validation in the automotive sector has been added: simulation. It is - in contrast to the classical test - completely IT-based. Simulation is increasingly used to replace time-consuming tests and cost-intensive physical prototypes. But at the moment it cannot replace testing completely. So the close cooperation of testing and simulation is decisive for a long-term competitive advantage. However, ways of thinking, data types, data structures and data storage are usually very different between tests and simulations. (Pischinger and Seiffer, 2016)

\subsection{Problem definition}

For over 100 years, the discipline of testing has developed independently. With increasing technical possibilities (sensor technology, measuring technology, IT, ...), growing requirements and increasing complexity of products, the number and variety of tasks, as well as the complexity of tests increased. This has led to higher data volumes and an increasing number of different data types. These are often very special and in some cases can only be limitedly evaluated and processed in automated manner. Test processes can be very different and their reproducibility (e.g. environmental and boundary conditions in real road tests) require statistical measures. Simulations, on the other hand, have clear, comprehensible processes, firmly defined environmental and boundary conditions and can be reproduced exactly. Data types are accurately defined (even if heterogeneous), data structures and data storage are clearly determined by IT support. In order to evaluate and improve the quality of a simulation, a comparison with the real test must take place.

In industrial practice, however, it often turns out that testing and simulation departments work separately from each other. Exchange of data and information is not automated and therefore a lot of time and information is lost in gaining the needed data or work has to be done repeatedly. Systems are getting more and more complex, an increasing number of people from different departments, companies or even countries work together on the same project. It has to be clear who needs which kind of data and information at what time during the development process. Access has to be granted to everyone who requires it - but also has to be limited to prevent misuse or overflow of data.

SPDM (Simulation Process and Data Management) systems are established in many companies, test process and data management systems are rarely used. An industrial use of a combination of both (in an interdisciplinary area) is not known to the author. It is therefore necessary to examine:

- What types of data and information are generated in testing and simulation and how are they stored or made available to others?

- Are there standard processes in testing and simulation which can be classified and adapted?

- How can test data management and simulation data management be combined?

\subsection{Definition of objectives}

In order to improve collaboration between testing and simulation departments this paper analyses verification and validation processes in the carriage development department of a motorcycle manufacturer. Types of data, data storage and data exchange, as well as the prevailing IT structure are examined in the use case. Test and simulation processes are mapped, standardised and classified. To support automation of data and information exchange recommendations are worked out to integrate test data management (TDM) and simulation data management (SDM). Therefore, different existing standards in TDM and SDM were analysed and evaluated. 


\section{STATE OF THE ART AND RELATED WORK}

In this paper the term "verification and validation procedures" is used to summarize all kind of actions to check if the product fulfils the requirements as well as if the requirements are suitable to satisfy the needs of the customer. (Deutsches Institut für Normung (DIN), 2015) This contains implementation verification, specification verification, conceptual model validation, operational (results) validation as well as theory validation. (Sargent, 2013)

Collaboration in product development is often described only on a very generic level. Some examples can be found in Ehrlenspiel and Meerkamm (2013), Pahl et al. (2007), VDI (1993, 2004). Luft et al. (2013) gives a good overview of different generic process models in product development over the last 25 years. Krehmer et al. (2011) extended existing generic models and added more detail in order to monitor the maturity of the product during the whole development process.

More detailed analyses of the collaboration between design and simulation departments have been made in the institute of Professor Lindemann (e.g. Herfeld et al. (2006), Kreimeyer et al. (2006), Maier et al. (2011), Schweigert et al. (2016)).

In this paper the collaboration between testing and simulation departments is analysed. Therefore a few abbreviations and standards in test and simulation data management are explained in the following sections:

\subsection{SPDM}

While Product Data Management (PDM) systems initially mainly stored data from Computer Aided Design (CAD), Product Lifecycle Management (PLM) nowadays organizes all data belonging to the product over the entire product lifecycle in a database. In Simulation Data Management (SDM), all data belonging to the simulation (e.g. input decks, results, evaluation, ...) are managed. Measurement Data Management (MDM) systems manage measurement data from experiments or tests.

Simulation Process and Data Management systems (SPDM) not only store data but also enable the support of the entire simulation process. These systems link the inputs and outputs of pre-processing, solving and post-processing programs and improve, document and automate the simulation process. They enable process traceability, rights management, easy calculation of variants and automation of simulations as well as reporting. (Pischinger and Seiffer, 2016)

\subsection{ASAM-ODS}

The Association for Standardisation of Automation and Measuring Systems (ASAM) is an interest group for standardisation formed by various manufacturers and service providers as well as research organisations. It issues different standards for test environments or automation of test processes. The standard ODS (Open Data Services) is a data model for test data that ensures a consistent storage and movement of data not depending underlying hardware. Data model, interfaces, physical storage and transport format are clearly defined. A basic model exists in which each piece of information (e.g. component, test equipment, boundary conditions, unit, ...) has a fixed place. This is concretized in an application model in each case. The output format is ATFX (ASAM Transport Format). (Rapf, 2016)

\section{3 openMDM}

openMDM is based on the ASAM-ODS standard. It is a generic ASAM ODS application model which focuses on the automotive industry. It offers a basis for client programs through an extended programming interface. openMDM includes not only data storage and data transfer (like ASAMODS), but also the test process itself, as well as its preparation. It supports the design of experiments. Different openMDM clients exist from several companies. These usually include a navigator and a search function, but also simple output features such as a chart viewer or export functions to other formats. Only test preparation, test execution and data processing take place outside the openMDM system. (Materna, 2010; Rapf, 2017; Rapf and Schwarzbach, 2016; Wittek, 2017)

\subsection{ISO-MME}

The ISO-MME standard (more precisely: ISO TS 13499) was first published in 1998 and standardizes generated data in frontal crash tests with vehicles in order to simplify data exchange. The main format of the standard are text documents in ASCII (American Standard Code for Information Interchange), but images and videos can also be transmitted. A clear data structure and principles such as 
compliance with SI units or zero values are defined. Compared to ASAM-ODS and openMDM, it has a relatively simple file structure. Explanations, diagrams and additional information form an important part of the standard. In principle, the standard only specifies the structure, but it is supported by many programs and so is still relevant today as an exchange format. (ISO, 2014a)

\subsection{STEP AP209e2}

STEP (Standard for the exchange of product model data) is divided into various application protocols (APs). All APs are supervised by the ProSTEP iViP Association in Germany and PDES, Inc. in the USA. The application protocol AP209e2 (also known as ISO 10303) is based on the protocol AP242, which aims to standardize the Business Object Model (BOM). It connects properties which are not geometric, such as project structure, document structure, metadata or kinetics. It's main goal is to connect SDM, PDM, CAE and CAD via a common exchange format. The model - which completely includes the AP242 protocol - is thus able to handle almost any application of data exchange between different simulation programs. (ISO, 2014b; ProSTEPiViP, n.d.)

\subsection{SimPDM}

SimPDM is a recommendation for automotive industry further developed from STEP AP209 and was published by the German Association of the Automotive Industry (VDA) as standard VDA 4967. It contains many basic ideas of STEP AP209, but is different in content. SimPDM shows how data structures should be set up and handled in order to integrate simulation data into PDM systems. A metadata model is stored on the macro level of the xDM system, which allows access to data stored in the micro level. Metadata include e.g. author, date of creation, date of release, file path and production status. The metadata model describes relationships between metadata of individual files created by the different systems in the management system. (Anderl and Malzacher, 2009; VDA Arbeitskreis PLM, 2008)

\subsection{Comparison of standards in simulation and measurement data management}

The standards ASAM ODS, openMDM, ISO MME, STEP AP209e2 and SimPDM were compared based on their suitability for testing or simulation, the area in which they are applicable, and the type of connectivity. A triple scale with "good", "partial" and "poor" was used for the evaluation. All of the examined standards are only rated "good" in the subject suitability in either testing or simulation. No standard has been examined which fitted "good" in testing and simulation. Some standards are applicable for all kind of tests or simulations, others are originally created for automotive industry (e.g. openMDM, ISO MME) but can be extended. Connectivity could be via ATF, ATFX, CORBA, REST or only via file transfer - depending on the standard. More details and an overview of the results can be found in Schönwald, Vahrenhorst et al. (2018).

\section{METHOD}

In order to answer the research questions mentioned above, a literature research on test and simulation data management was carried out first. The Karlsruhe Virtual Catalogue, Google (www.google.de) and the databases SCOPUS (www.Scopus.com), EMERALD (www.emeraldin-sight.com) and WEB OF SCIENCE (www.webofknowledge.com) were used. It was searched for e. g. testing, simulation, measurement, data management, process, Systems Engineering, Computer Aided Engineering, Computer Aided Testing, experiment, CAT CAE cooperation, collaboration, test data, simulation data, openMDM, ASAM ODS, SimPDM, STEP, ISO MME, SPDM, ... and all kind of combination of these keywords.

An overview of generic test types was created and those were assigned classifiers. The purpose of these classifiers is to arrange test data in test data management and thus make them more easily accessible for later tests.

Existing IT systems and established standards from the areas of test and simulation data management were analysed and compared. In order to evaluate the validity and timeliness of the results, two semistructured expert interviews were conducted with engineers from an SPDM system manufacturer.

In a preceding study of the author (Schönwald, Fleskes et al., 2018) a method was presented and evaluated to analyse data and information flows in product development in detail. Some results were BMPN (Business Process Model and Notation) diagrams, Design Structure Matrices or Domain 
Mapping Matrices of verification and validation procedures in the development department of a motorcycle manufacturer. For this paper they were analysed and combined to one generic test process and one generic simulation process.

In order to deepen the findings and ideas from the literature research using a practical example and to check their feasibility, 20 semi-structured expert interviews were conducted with test and simulation engineers in the same motorcycle company. It was e. g. asked, what kind of tests or simulations they do, to describe the different processes, what kind of data and information they need, with whom they work together, how they evaluate and store their data, ... All tests currently used in the carriage department were analysed and grouped according to existing classifiers. The prevailing data storage as well as the types and size of the data and information packages were also examined in detail. For this purpose, internal drives were searched, existing overviews and lists were extended and merged. Subsequently, the results of the expert interviews were compared with the findings of the literature research and suggestions for the integration of test and simulation data management were developed. One approach was chosen and is now being implemented into the prevailing IT-structure of the motorcycle company.

\section{USE CASE: CARRIAGE DEVELOPMENT DEPARTMENT OF A MOTORCYCLE MANUFACTURER}

\subsection{Data and information flows in testing}

Test procedures and approaches often reflect early experiences of test engineers instead of orienting themselves on standardized procedures. No uniform classification of known test methods has been found in literature. An overview of the 33 test types generically described by Engel (Engel, 2010) was compiled and each one described with the following 6 classifiers: test case, test scope, test environment, test interpretation, time of test and test data type. More details can be found in (Schönwald, Vahrenhorst et al., 2018).

The carriage development department of the analysed motorcycle manufacturer carries out approx. 300 different tests in the areas of environmental testing, vibration, tension/pressure and force measurement, endurance tests, extended use test, misuse and special events.

To classify these different tests, the following criteria have been used: name, field, parts scope, properties, type of test and test environment.

The three main goals of these tests are:

- Determine material parameters that cannot be derived from purely analytical observations.

- Verify development results whereby specific requirements to the technical system that is to be developed are proven.

- Validate simulations in order to show that this simulation produces sufficiently accurate calculation results.

In our case the validation of simulations is very interesting because a lot of potential for improvement has been found.

The design of experiments is mainly carried out with the help of Excel lists and Word documents. A partial automation for the creation of standardised test checklists is currently being set up using SharePoint. Data storage takes place on internal drives. The structuring and naming of files is different in the different departments, but also within the individual groups - depending on the individual engineer or certain projects - variances occur. This sometimes leads to multiple storage or empty folders. With derivative developments, results from predecessor projects are used in some areas - but references to them are usually missing. No cross-departmental naming convention is used for data storage.

Not all tests are carried out internally, some are outsourced. The data exchange with external service providers in the field of testing is mainly carried out via e-mail. Several thousand files are created per vehicle project. The data volume is very different and varies between the projects in a range between $500 \mathrm{MB}$ and $100 \mathrm{~GB}$.

Test results are almost always presented in text form (e.g. *.docx, *.pdf) and often supplemented by photos (e.g. *.jpeg) and videos (e.g. *.mov, *.avi). More rarely, other formats (e.g. ASCII code) or diagrams for force values or drag coefficients are saved. 
On a generic level all those 300 tests have almost the same structure (see Figure 1). They are triggered by a test order which is evaluated and objectives are set. Based on the objective pursued with the test, the next step is to concretize the test artefacts, determine test cases (in the sense of load cases) as well as model assumptions and prepare the test environment, i.e. the actual test bench and the measurement data acquisition for the test. The tests must then be carried out under the required test conditions taking into account that statistically meaningful results have to be obtained. The measured test data is stored and interpreted. The result of the test is a report which can then be played back into the development process. There are different quality checks which lead to loops in the process. This generic procedure can be concretized with the help of the classifiers (see above) for certain tests.

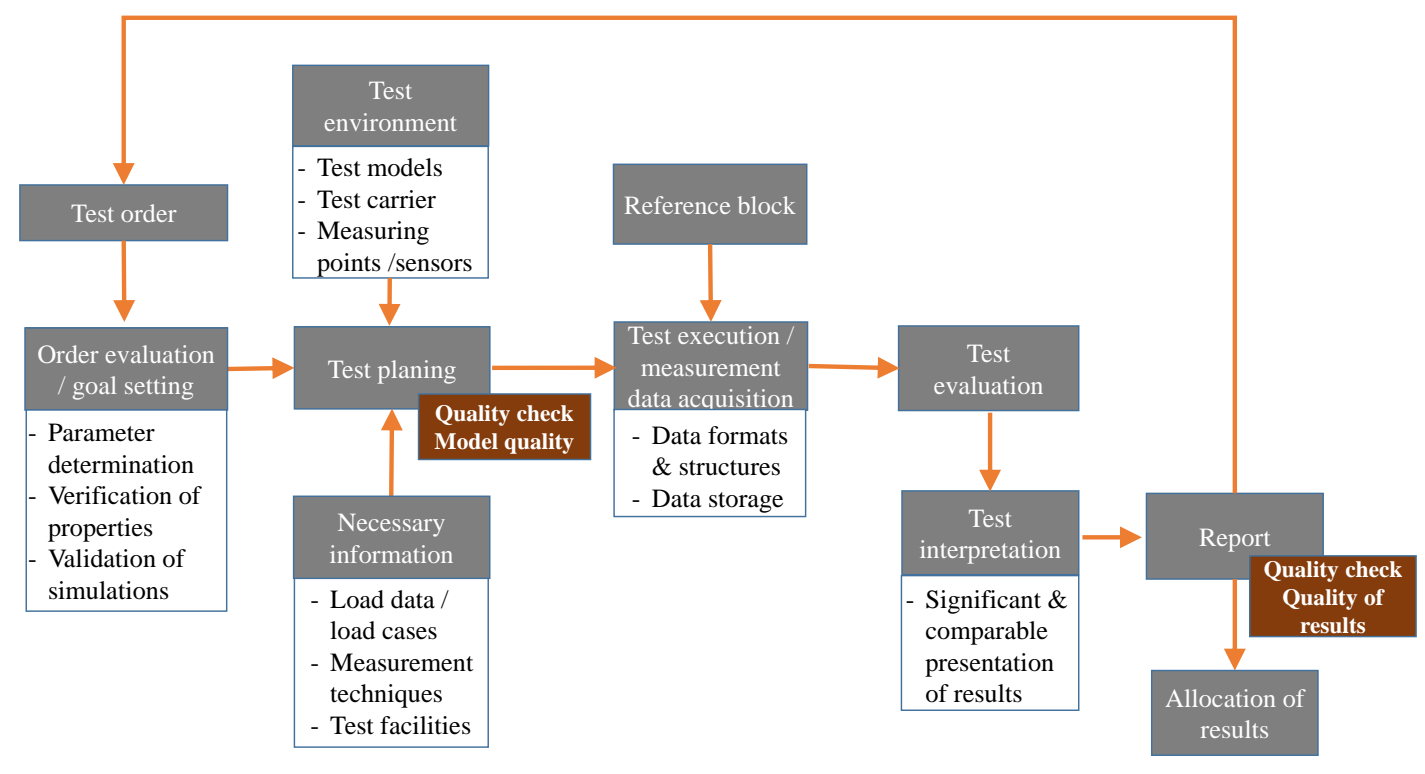

Figure 1. Standard test process

\subsection{Data and information flows in simulation}

In our use case about 60 simulations are associated with the 300 tests. All simulations mainly follow the same linear process consisting of the steps of input, preprocessing, solving, postprocessing and output (see Figure 2). This generic process can be adapted by variating e.g. load cases, load data, references, ...

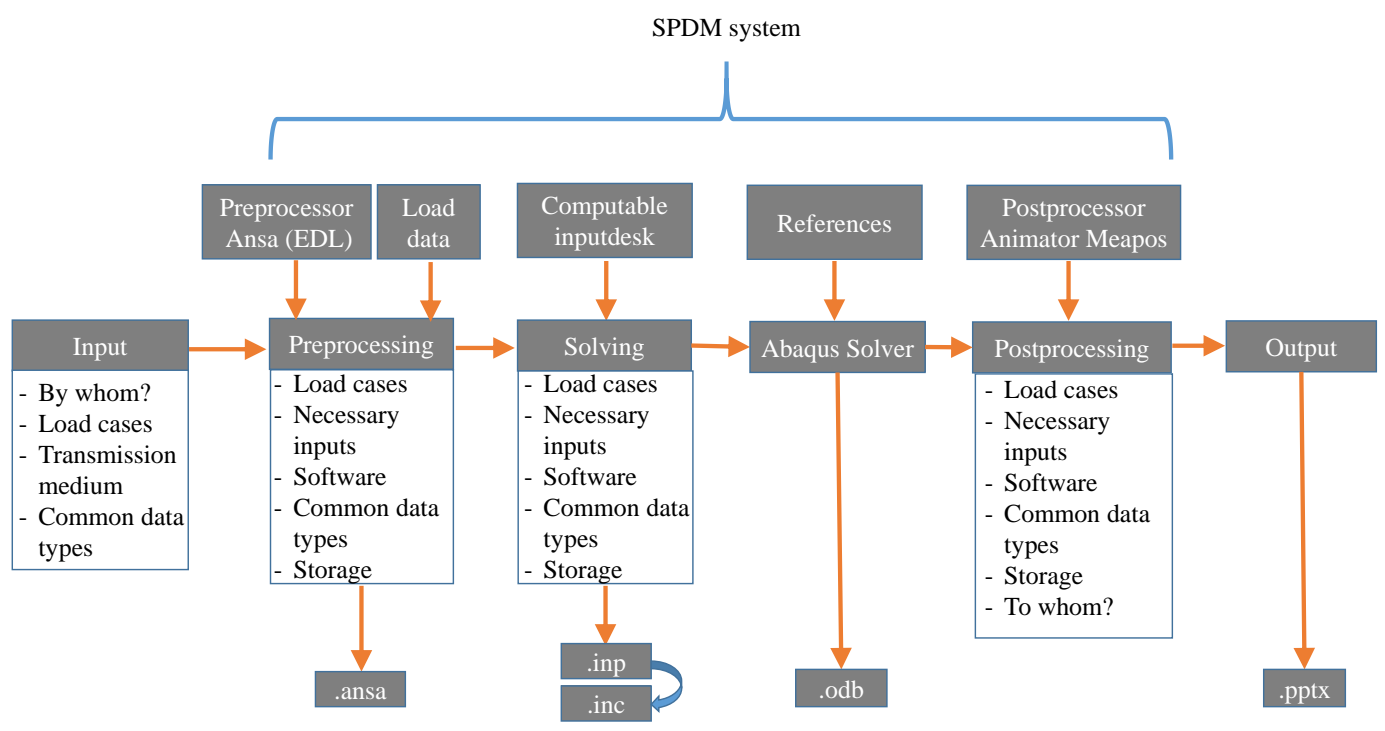

Figure 2. Standard simulation process 
Simulations are mainly carried out externally, simulation planning and data exchange are mainly carried out via an SPDM system or a cloud. For each simulation there are clearly defined modelling guidelines and calculation information templates, which can be accessed both internally and externally via a SharePoint solution. The reports are stored on an internal drive in addition to the SPDM system. The type of output files is i.a. *.ansa, *.inp, *.inc., *.odb and *.pptx.

\section{CONCLUSIONS FORM THE USE CASE}

\subsection{Comparison between testing and simulation processes}

In general simulation processes have a good reproducibility because they always use the same boundary conditions. Parameters can be variated very easily and special conditions can be simulated without major problems. The time and cost expenditure of simulations is moderate. Inaccuracies can occur due to modelling and used algorithms as well as ideal model assumptions.

Test processes on the other hand have a restricted reproducibility because they depend on boundary conditions (which are not always directly influenceable). Tests usually demand much time and costs because prototypes are necessary. Inaccuracies can occur due to sensors and measurement data recording. Variances in boundary conditions require statistical evaluation.

Data and information flows as well as type and structure of data and information are different in tests and simulations. An adapted structuring of workbenches which is not just based on interface definitions is needed.

In the use case the structuring of data storage and the use of standardized templates and processes is not only due to the use of an SPDM application - significantly better in the area of simulation than in the area of testing. Test data storage can be improved. The categorization and semi-automation of the test planning using SharePoint is a good approach, but does not allow the storage of test data due to limited storage capacity. This should only be seen as an intermediate step for the introduction of test data management.

The most serious challenges in the use case are:

- The existence of different forms of data storage (centrally via PLM, department-driven, projectdriven, locally on individual computers)

- The usage of different forms of information processing (reports with different structures, varying contents)

- The generation of a very large amount of data with each test (central administration of all test data is often not effective)

All in all, three database systems are used for data and information storage:

- The central storage of CAD data takes place in the PDM system.

- The storage of the data, which originates from the simulations and is further used in this context, takes place via the simulation workbench.

- Measurement data is partially stored via openMDM.

However, a large part of the test data is stored on file servers in simple folder structures - often different in the individual departments and working groups.

\subsection{Integration of test and simulation data management}

When implementing a consistent TDM, it is therefore important to link these data-managing IT tools via the process description in such a way that the stringent flow of data and information is guaranteed. In the sense of a continuous data protection management, it is also necessary to coordinate and merge the dependencies of the data and information flows between TDM and SDM in the sense of the objective of the verification or validation procedure. Both must be integrated at IT level. For this some standards are offered. These standards were generally developed with the focus either on SDM or on TDM, a coupling of both is often only rudimentarily considered, whereby the individual activities are more or less well supported in the protection. 
Three categories of interfaces are to be distinguished for this purpose:

- Process interfaces are to be concretized from the logical sequence of process steps and individual activities.

- Organizational interfaces define responsibilities for the process steps and are accordingly connected with release mechanisms, rules for data and information transfer, etc., which support quality assurance.

- Formal interfaces must be considered in order to secure continuous data and information flows via existing IT tools.

The reviewed standards (see 2.1 to 2.7 ) support either test data management or simulation data management. All standards cover the field of the considered use case. The connectivity of the standards can only partly be classified as "good". The following 5 options would be conceivable to support the integration of test and simulation data management (see Figure 3):

Coupling SDM application and TDM application using

- Ontologies specific to application pairs

- Two object-oriented interfaces

- A higher-level, intermediary application

- Use of a common - possibly newly created - standard

- Adaptation and integration of one standard into another standard
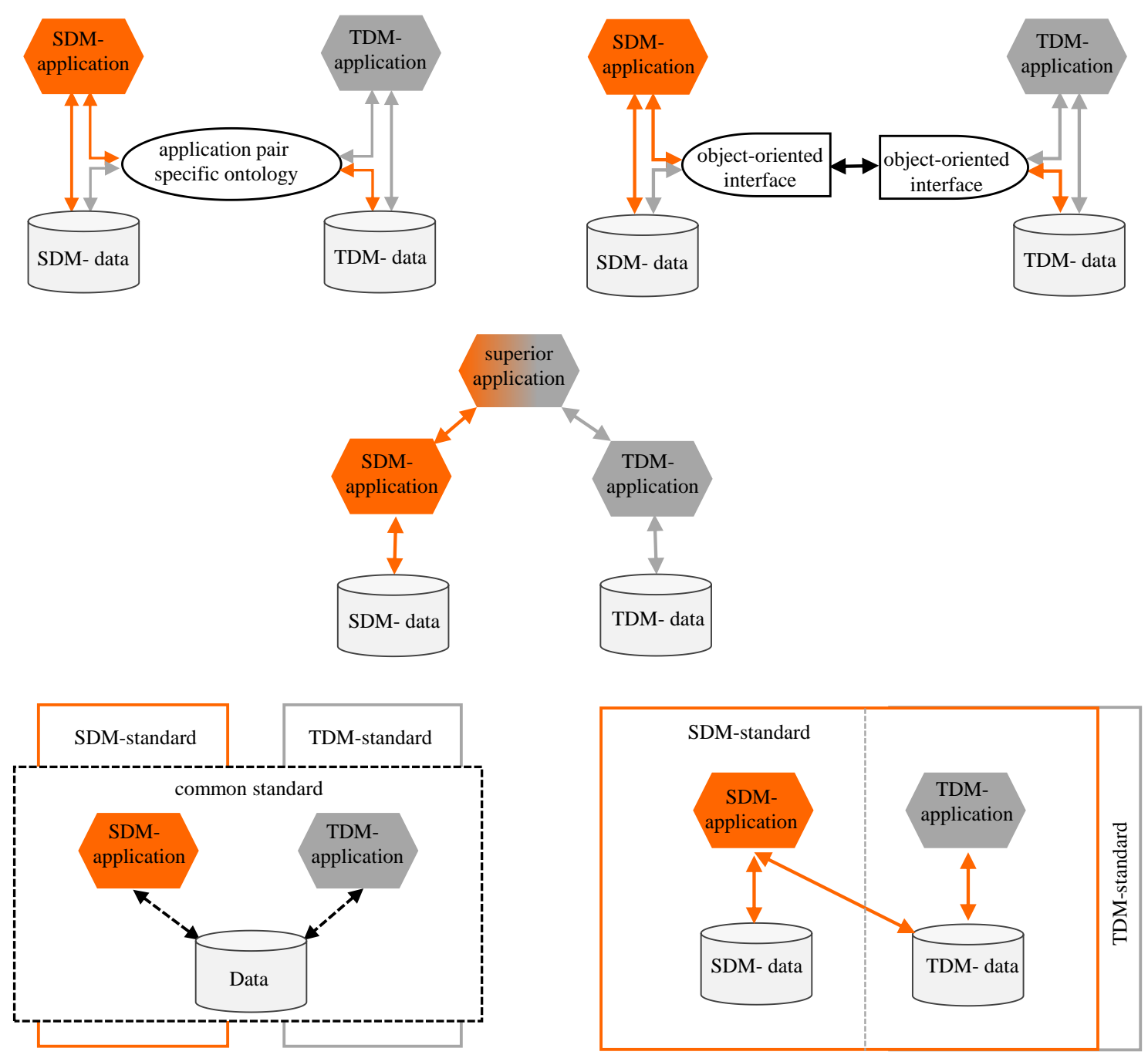

Figure 3. Approaches to integrate test data and simulation data management 
It has to be discussed for each case, which of these approaches fits best to the existing IT-Structure of the company. In our use case we decided to integrate test data via ISO-MME Standard into an existing SPDM system. This is a very easy and practical approach, but most of the work has to be carried out manually at the first implementation. Pros and cons of each approach, problems and potential as well as the evaluation of the implementation will be described in another publication.

\section{CONCLUSION AND OUTLOOK}

Collaboration and automation, as well as joint data and information storage in the areas of testing and simulation, hold significant optimization potential. Therefore, different standards in SDM and TDM were analysed and evaluated. Generic test processes in literature were examined and categorized with classifiers. In the carriage development department of a motorcycle manufacturer verification and validation processes were analysed in detail and standard test and simulation processes established. The prevailing data storage and IT structure in testing and simulation departments of the use case were examined.

In simulation data management there are good (also commercially available) solutions, but these are in most cases only partially compatible with test data management. Various approaches have been shown for the integration of test and simulation data management. All of them are conceivable but the prevailing processes and IT structure in the companies have to be analysed and then be decided which option fits best.

However, not only questions of a technical nature but also of a non-technical nature should be considered to improve efficiency and effectivity in verification and validation procedures. Communication, cooperation, coordination and collaboration between all participants of the product development process has to be supported and managed. It has to be analysed, documented, maintained and provided who needs what kind of data and information at what time in which quality. Interfaces between testing and simulation departments with other areas e.g. design, requirements engineering, risk management or quality management are examined in the use case right now.

Different mind-sets and attitudes of all involved people have to be considered, problems and wishes should be examined more closely before implementing new processes or IT systems.

\section{REFERENCES}

Anderl, R. and Malzacher, J. (2009), "SimPDM - Simulationsdatenmanagement-Standard nach Maß”, CAD CAM, No. 1-2, pp. 38-41.

Deutsches Institut für Normung (DIN). (2015), DIN EN ISO 9001:2015 Qualitätsmanagementsysteme Anforderungen, DIN-Normenausschuss Qualitätsmanagement, Statistik und Zertifizierungsgrundlagen (NQSZ), Deutschland, p. 71.

Ehrlenspiel, K. and Meerkamm, H. (2013), "Integrierte Produktentwicklung - Denkabläufe, Methodeneinsatz, Zusammenarbeit, Konstruktion,” available at:https://doi.org/10.3139/9783446436275.

Engel, A. (2010), Verification, Validation, and Testing of Engineered Systems, In: Sage, A. (Hrsg.): Wiley Series in Systems Engineering and Management, 73rd ed., John Wiley \& Sons, New Jersey, available at: https://doi.org/10.1002/9780470618851.

Herfeld, U., Kreimeyer, M., Deubzer, F., Frank, T., Lindemann, U. and Knaust, U. (2006), “Verknüpfung von Komponenten und Funktionen zur Integration von Konstruktion und Simulation in der Karosserieentwicklung", VDI Berichte, No. 1967 I, pp. 259-276.

ISO. (2014a), ISO/TS 13499:2014 Road Vehicles - Multimedia Data Exchange Format for Impact Tests.

ISO. (2014b), ISO 10303-209:2014-12 Industrial Automation Systems and Integration - Product Data Representation and Exchange - Part 209: Application Protocol: Multidisciplinary Analysis and Design.

Krehmer, H., Meerkamm, H. and Wartzack, S. (2011), "Monitoring a property based product development from requirements to a mature product", Iced'11, No. August, pp. 1-11.

Kreimeyer, M., Herfeld, U., Deubzer, F. and Lindemann, U. (2006), "Effiziente Zusammenarbeit von Konstruktions- und Simulationsabteilungen in der Automobilindustrie", Competence in Design and Development, Vol. 02 No. 01, pp. 1-13.

Luft, T., Krehmer, H. and Wartzack, S. (2013), “An advanced procedure model for property - based product development", International Conference on Engineering Design, ICED13, No. August, pp. 1-10.

Maier, A.M., Doenmez, D., Hepperle, C., Kreimeyer, M., Lindemann, U. and Clarkson, P.J. (2011), "Improving Communication in Design: Recommendations from the Literature", 18th International Conference on Engineering Design, Impacting Society through Engineering Design (ICED 11), No. August.

Materna, R. (2010), OpenMDM - What's Behind?, Audi AG, Ingolstadt, pp. 1-17. 
Pahl, G., Beitz, W., Feldhusen, J. and Grote, K.-H. (2007), Engineering Design: A Systematic Approach, Springer, No. 2, p. 617.

Pischinger, S. and Seiffer, U. (2016), Vieweg Handbuch Kraftfahrzeugtechnik, 8. Auflage., Springer Vieweg Verlag, Wiesbaden, available at: https://doi.org/10.1007/978-3-8348-8298-1.

ProSTEPiViP. (n.d.), "STEP AP209 online”, available at: http://www.ap209.org (accessed 23 April 2018).

Rapf, D. (2016), "Standards im Datenmanagement ASAM ODS und openMDM”, ATZ Extra, Wiesbaden, May, pp. 32-39.

Rapf, D. (2017), “The openMDM roadmap The future of measured data management", Testing Expo Stuttgart 2017, pp. 1-21.

Rapf, D. and Schwarzbach, M. (2016), “openMDM ® - an Open Source Platform for Measured Data Management”, Automotive Diagnostics Systems Summit 2016, pp. 1-24.

Sargent, R.G. (2013), "Verification and validation of simulation models", Journal of Simulation, Vol. 7 No. 1 , pp. 12-24.

Schönwald, J., Fleskes, J., Forsteneichner, C. and Paetzold, K. (2018), “A method for a detailed analysis of verification and validation processes in product development”, International DESIGN Conference 2018, Dubrovnik, Croatia, pp. 1313-1324.

Schönwald, J., Vahrenhorst, D., Chojnacki, A., Zschako, S., Sundarp, L. and Paetzold, K. (2018), "Integration von Versuchs- und Simulationsdatenmanagement in der Produktentwicklung", DFX 2018: 29th Symposium on Design for X, München, pp. 107-118.

Schweigert, S., D'Albert, H. and Lindemann, U. (2016), "Enhancement of collaboration and communication between design and simulation departments by methods of requirements engineering", Proceedings of International Design Conference, DESIGN, Vol. DS 84, pp. 1397-1406.

VDA Arbeitskreis PLM. (2008), VDA 4967: Simulation Data Management - Integration of Simulation and Computation in a PDM- Environment (SimPDM), No. Version 2.0, Frankfurt.

VDI-Gesellschaft Entwicklung Konstruktion Vertrieb. (1993), VDI 2221: Methodik Zum Entwickeln Und Konstruieren Technischer Systeme Und Produkte, Deutschland, available at: https://doi.org/10.1017/CBO9781107415324.004.

VDI-Gesellschaft Entwicklung Konstruktion Vertrieb. (2004), VDI-Richtlinie 2206: Entwicklungsmethodik Für Mechatronische Systeme, Beuth Verlag, p. 118.

Wittek, A. (2017), Setup Development Environment for the OpenMDM ( R ) Application Eclipse Mdm.

\section{ACKNOWLEDGEMENT}

The work on this paper is part of the research project "Management of verification and validation procedures in product development" (AbsManPE) which is funded by the Bavarian Research Foundation (BFS). The BFS and the involved industry partners are warmly thanked for their support. 